\title{
BMJ Open Quality Improving handover between triage and locality wards in a large mental health trust
}

\author{
Alice Debelle, Mark Dashwood, Louisa Bird, Ranga Rao, Thomas Reilly
}

To cite: Debelle A, Dashwood M, Bird L, et al. Improving handover between triage and locality wards in a large mental health trust.BMJ Open Quality 2017;6:e00023. doi:10.1136/ bmjoq-2017-000023

- Additional material is published online only. To view please visit the journal online (http://dx.doi.org/10.1136/ bmjoq-2017-000023).

Received 14 February 2017 Revised 9 October 2017 Accepted 10 October 2017

\section{CrossMark}

South London and Maudsley NHS Foundation Trust, London, UK

Correspondence to Dr Alice Debelle, South London and Maudsley NHS Foundation Trust, London, UK: alicedebelle@doctors.org.uk

\section{ABSTRACT}

Background South London and Maudsley NHS Foundation Trust is the largest mental health trust in the UK, serving four boroughs in South East London. In 2014, the 'triage ward' system was introduced in three boroughs. Similar to an acute medical admission unit, the triage ward would rapidly assess and treat all new admissions. The patients would either be discharged or admitted to a 'locality ward' for further treatment.

Problem The unforeseen consequences of the 'triage ward' system were duplications and omissions of medical tasks on receiving wards, which affected efficiency and quality of care. This was due to a lack of formal medical handover. We aimed to improve efficiency and patient safety by formalising the junior doctor handover between triage and locality wards, ensuring every patient transferred had a documented handover in their electronic notes.

Method We consulted our colleagues with a survey, ascertaining their views on the current system, the need for a more formalised system and what form that system should take. Using their feedback, we devised a handover template, to be completed for all patients transferred to locality wards. We then rolled the project out to the other two boroughs using the same methodology.

Results A follow-up survey showed improvement in our baseline results and that the majority of transferred patients were formally handed over. Serious incident data showed a decrease in incident rates pre-intervention and post-intervention. The intervention was sustained a year later. The transfer of the intervention to other sites was problematic.

Discussion The project showed the lack of handover was a concern shared by colleagues, and they considered our template a useful way of addressing this. The results suggested that the intervention was sustainable despite frequent rotations of staff. The difficulties in transferring an intervention to new sites are discussed.

\section{PROBLEM}

In 2014, the 'triage ward' system was introduced into three boroughs (Lewisham, Lambeth and Croydon) in South London and Maudsley (SLaM) NHS Foundation Trust. Similar to an acute medical admission unit, the triage ward would rapidly assess and treat all new admissions. The patients would either be discharged or admitted to a 'locality ward' for further treatment. Patients transferred from triage to locality wards were not handed over, although a few with pressing issues may be discussed informally through emails, phone calls or ad hoc face-to-face meetings. This leads to potential problems. In order to identify which tasks were outstanding, the receiving medical team needed to read through electronic records which was time consuming. Often it was unclear from these records which tasks were complete and which were pending; thus, tasks and referrals could be missed or duplicated. More than once, a referral to another specialty was duplicated due to inadequate handover. Physical health issues were not immediately apparent. For example, a patient with poorly controlled diabetes may not have optimal blood glucose monitoring as planned. Overall, the lack of handover impaired efficiency, patient safety and overall quality of care. In addition, patients were often transferred out of hours, and so, the necessary phone call or email to handover important information could be delayed or not made at all.

We aimed to improve efficiency, patient safety and overall quality of care by formalising the medical handover between triage and locality wards, by developing a system whereby a written succinct and pertinent handover was provided for every patient transferred, over a 4-month period. After piloting the project at the Lewisham site, a secondary aim was then to expand the project to the two other boroughs, Croydon and Lambeth, which were using the triage model.

\section{BACKGROUND}

The project was based in SLaM NHS Foundation Trust which provides the widest range of NHS mental health services in the UK. Overall, the trust serves a local population of 1.3 million people in some of the most deprived areas of London.

For the past decade, the importance of safe handover has become ever more salient as the European Working Time Directive and 
resultant shift patterns increase the frequency of transfer of care between teams. The British Medical Association in collaboration with the National Patient Safety Agency, the General Medical Council, the Royal College of Physicians and the Royal College of Surgeons have all released expansive guidance on handover. ${ }^{1-4}$

Handover recommendations from these bodies and related Quality Improvement (QI) projects tend to focus on improving handover between shifts. ${ }^{56}$ There has been less focus on handover of transferred patients between medical admission units (MAUs) and wards for instance, or from intensive therapy units to wards, although some QI projects have begun to address this. ${ }^{78}$ With mounting bed pressures, patients are being transferred between acute units and wards out of hours, often without the responsible doctor's knowledge. ${ }^{78}$

In psychiatry, this has traditionally been less of a problem, as patients are usually admitted to a ward in which they remain until discharge. However, at SLaM NHS Foundation Trust, a triage system was introduced, in an attempt to improve efficiency of inpatient care. ${ }^{9}$ The Lewisham Triage system started in 2003, Lambeth in 2012 and Croydon in 2014. In this system, all acute admissions are directed to one triage ward that has a capped length of stay (7-10 days). Similar to an MAU, the aim is rapid stabilisation of the acute episode and either discharge from triage ward or transfer to a locality ward. ${ }^{9}$ For Lewisham and Lambeth, there are 900-1000 admissions a year, $50 \%$ are discharged within 7 days and the rest transferred for a longer stay. ${ }^{9}$ Again, like MAU, patients from these triage wards are often being transferred out of hours and to date, without any formal handover process between doctors in place.

Patients with serious mental illness often have complex physical health needs and an improper handover "can be a major contributory factor to subsequent error and harm to patients'. ${ }^{1}$ This project aimed to address the lack of medical handover, an unforeseen consequence of the introduction of the triage ward system in psychiatry.

\section{BASELINE MEASUREMENT}

We used a short survey to measure junior doctors' opinions on the current state of handover before our intervention. We repeated the measure 4 months after implementing change. To avoid potential confounders, the same doctors were surveyed before and after.

The survey was anonymous, so it should be a valid reflection of doctors' opinions. There were no major changes in the transfer system other than our intervention while the project was underway, so it would be very unlikely that changes in survey results from baseline could be due to much else except our intervention. The rationale for choosing this measure was that our intervention was solely for junior doctor use, so the perceived utility of our intervention for these stakeholders was important. While it was anticipated that patient outcomes would be improved if key medical information was handed over, it was difficult to find a way of capturing this and attributing any improvement directly to the handover system. Serious incident (SI) data were collected preintervention and postintervention, but much of this data was for incidents not related to the handover: for example, violence, absconding and staffing issues. This limitation is discussed below.

The survey questions were as follows:

How adequate do you think the current handover system is overall?

How important is it to have a handover system?

Currently, how easy is it to ascertain which jobs are outstanding for patients transferred from triage ward?

What form do you think the handover should take? Options: verbal, email, brief entry on electronic notes, brief discharge summary on electronic notes

Do you have any suggestions for how the handover process could be improved?

We asked doctors to answer the first three questions using 1-5 Likert scales, with one being the lowest rating.

The results of the baseline measurements for all three boroughs are displayed in the attachment.

We obtained SI data 6 months preintervention as a baseline measurement and compared this with data 6 months following the intervention. This was used to monitor changes in patient outcomes.

The baseline results showed that doctors on the locality wards found the current handover system inadequate. They found it difficult to find the information they needed and thought a brief entry on our electronic records (electronic patient journey, ePJS) would be the best format. The vast majority of doctors thought it was important to formalise handover. See online supplementary file: baseline result.

\section{DESIGN}

During the pilot project in Lewisham, the baseline measurement survey formed our main consultation process, and using these results, we came to the final design for our intervention, a formal documented handover on ePJS, example below:

Triage Junior Doctor handover:

- Outstanding jobs: has refused bloods, physical, ECG, so these are still pending

- Referrals: neuropsychiatry referral complete 4/7/14-see correspondence, needs to be chased

- Physical health problems: type 2 diabetic on metformin, blood sugars stable

- Other comments: nil

We concluded the above would be the most effective handover system for two reasons. First, it is the format that the majority of the doctors thought would be the most helpful (see Baseline measurements section). Second, it had a consistent format that would convey the important information succinctly and clearly. To ensure the workload of triage doctors was not significantly increased, we included only the most pertinent information. 
The main problem we anticipated at this stage was that patients could be transferred without our knowledge (ie, not 'captured') and thus not receive a handover.

A simple strategy was devised to increase 'capture rates', whereby each evening, a doctor on triage ward would write down a list of the current inpatients on the ward. The next morning this list would be compared with the updated patient list on the ward whiteboard. The patients no longer on the ward had a handover duly written.

Given the only marginal increase in workload, we felt the intervention would likely be sustainable over the long term. To ensure continuity between 6 monthly rotations of doctors, we incorporated the handover system into the 'Junior Doctor's Guide' for Lewisham triage ward, so that it became an expected part of the junior doctors routine work. We also involved the triage ward consultant (RR) who was similarly committed to sustaining the project across rotations.

\section{STRATEGY}

\section{Plan Do Study Act (PDSA) cycle 1}

We planned the intervention as above. The doctors on triage ward wrote down the list of patients each evening and compared it with the ward whiteboard the next morning, noting patients that were transferred and completed the handover template for those patients. After 4 months, we repeated our baseline measure survey and examined the 'capture rates'; the proportion of transferred patients that had a handover in their records during that period. We chose 4 months as an appropriate length of time as it would give us a representative picture of capture rates allowing for fluctuations in staffing levels and personnel (eg, permanent staff vs locum doctors), level of activity on triage ward, peaks and troughs in transfer rates and other arbitrary variations.

During the first 4-month study period of the intervention, it became apparent that there was variation in the brevity and content of the handover between the doctors working on triage ward. This was due to a lack of clarity among our colleagues, and our key learning from this was we needed to communicate to all our medical colleagues, including locum doctors, what the aim of the handover was. Within our resurvey results, there was clear improvement in ratings of the handover process. There were suggestions, however, regarding inclusion of information regarding Mental Health Act (MHA), tribunal reports status and tribunal dates. While capture rates were very good, not all patients had received a handover during this first cycle, and having studied these individual cases, it appears that it was either when a locum doctor was covering the ward and not familiar with the process or when a patient had arrived and been transferred out of hours, so it was impossible for the ward doctor to know the patient had been on the ward. We presented at a Trust Safety Conference, and following further engagement with stakeholders in other hospitals, it was agreed to roll out the project to the other two boroughs.
We identified areas for improvement in our intervention from the first PDSA cycle:

- further standardisation of the handover so that it fulfilled its aims of being succinct and pertinent,

- adding a heading for MHA information,

- increasing the capture rates further, and

- rolling out the project to other boroughs.

\section{PDSA cycle 2}

The key learning from this cycle was communication; so for the second cycle, we worked to standardise the handover by simply speaking to our colleagues on triage informally and showing them examples so that they fully understood what was required and the purpose of the intervention. We included the example in the Junior Doctor's Guide. We enlisted the support of our senior colleagues who were then able to draw locum doctor's attention to the handover system, in order to increase capture rates further. We also took on board the suggestions from the survey and added an MHA section to our template.

We proposed the project to two junior doctor colleagues who were currently working on triage wards in Croydon and Lambeth. They then replicated the first PDSA cycle with baseline measurement, intervention, resurvey and measurement of capture rates. In Lambeth, it proved more difficult to gain adequate capture rates. The doctor implementing the change (MD) named the following reasons: difficulty in establishing when patients had been discharged from the ward. The method used in Lewisham generally transferred well to Lambeth, although it was more difficult if the patient list was not up to date. Monday mornings were problematic, as there was often numerous transfers over the weekend. Also, not all doctors on the ward were fully on board with completing the handover entries. There were often staffing issues on this busy ward and even small increases in workload were potentially unpalatable. When the author MD was not working on the ward, handover entries were less likely to be completed. In Croydon, there were no staffing issues and no locum doctors, so all three junior doctors were on board and aware of the project and were involved in its implementation. They also used an 'admissions book' that was already in place to keep track of transferred patients. This may explain the differences in capture rates between Croydon and Lambeth, although the survey results are similar and both show improvement in doctor's ratings.

The key learning from this second cycle was that the handover needed to become a standard part of the junior doctors' daily work, and for this to happen, it needed to be made official and incorporated into the daily routine. In Lewisham, this was via the Junior Doctor's Guide and senior support; in Croydon, this was by adding it on to a system that was already in place (the admissions book).

In addition to these two PDSA cycles, we assessed capture rates again in Lewisham, a full year after initiating our first cycle, to test sustainability of the project. Having enlisted the support of senior colleagues who helpfully 
informed locum doctors about the handover process, the capture rates had increased further.

\section{RESULTS}

Following baseline measurements, two outcomes were measured following implementation of the handover: (1) resurvey results and (2) capture rates.

We repeated outcome 2 in Lewisham a year after the second cycle, to test the sustainability of the project. Unfortunately, Croydon triage ward closed before we could test sustainability. The capture rate was $0 \%$ a year later in Lambeth as the project had not been sustained.

Overall, there was a clear improvement across all three boroughs for outcome 1; see online supplementary file. There was significant variation in the number of doctors surveyed between boroughs.

For outcome 2, capture rates were good in Lewisham and Croydon, less so in Lambeth: in Lewisham, of 128 transfers, $116(90 \%)$ had handovers (April-August 2015); in Croydon, of 20 transfers, $20(100 \%)$ had handovers (September-January 2016); in Lambeth, of 22 transfers, $10(45.4 \%)$ had handovers (March-April 2016.) The data set for Lambeth is incomplete, as the audit period was only 21 days, rather than 4 months.

Following refinements in Lewisham to improve capture rates as described above, an improvement was seen 1 year on, demonstrating sustainability. Between April and August 2016, of 122 eligible transfers, 112 (91.8\%) patients had handovers.

In terms of patient outcomes, number of incidents in SI categories that could potentially be affected by the intervention (such as clinical care) did reduce 6 months postintervention, compared with baseline measurement. See online supplementary file: results.

\section{LESSONS AND LIMITATIONS}

The notable strength of the project was its simplicity and ease of implementation, which resulted in improvement in the measured outcomes across all three boroughs and also contributed to its sustainability in the pilot borough. We achieved sustainability in Lewisham by incorporating the handover into systems that were already in place, so that it became a natural part of the junior doctors' daily work. We also used relationships with permanent staff to promote this. These strategies proved successful. Using the survey as both a consultation device and as a baseline measure meant that the stakeholders, the junior doctors themselves, had agreed on its intrinsic worth and utility prior to implementation; there was an appetite for this intervention. Thus, the receiving doctors expected and benefited from the handover, which motivated the doctors on triage to ensure it was completed.

The main challenge and limitations of the project came after the roll-out to other boroughs, and it showed how even a very simple intervention is difficult to replicate between sites. For example, at the time of the project in Croydon, there were far less transfers than other sites due to ward closures, so it did not give an accurate picture of how the implementation would work when the system was running as normal. This unforeseen factor gave a very small sample size, which is a limitation of the project. The ward subsequently closed prior to sustainability data being collected at 1 year.

In Lambeth, the project was not sustained after 1 year, where it was challenging to successfully implement the handover due to a number of human factors. The handover was only taken up by one of the doctors working on the ward, and therefore, data were only obtained over a 3-week period rather than 4 months. A possible reason for the low uptake among the junior doctors could be related to the additional work required in completing the handover entry. This is despite the general consensus that having a handover system would be helpful and that it was quick to complete. It has been observed in other quality improvement projects that changing the handover culture of departments can be challenging particularly when a handover culture is deeply embedded ${ }^{10}$; the changes are perceived as more time consuming than the current system in place ${ }^{11}$ or thought to involve additional work. ${ }^{12}$

One way to improve the implementation of the handover project would be to ensure that all the key team members were fully behind the project and finding ways to motivate them. If we were to do the project again, we would ensure all the doctors on Lambeth triage were directly involved in the surveying and consultation process, so that they would be more likely to realise the worth of the project and be accountable to the junior doctors expecting a handover. We would also attempt to involve the triage ward consultant which may help with encouraging the trainees to adopt the new handover system. The importance of engaging all the key team members, particularly senior staff, has been identified in other handover quality improvement projects. ${ }^{1013}$

Another limitation was capturing improvement in patient outcomes as a result of the intervention. SI data offer a broad snapshot preintervention and postintervention, but given the complex nature of ward life, it would seem impossible to ascertain how improvement in patient outcomes could be directly attributed to a better handover, which itself covers multiple aspects of patients' care.

In terms of the limits of generalisability, we have shown that the project was applicable to other boroughs within the trust, but more work is needed to make it as successful as the Lewisham pilot. The handover template was tailored to the specific needs of a psychiatric junior doctor, receiving a patient from the SLaM triage ward system, and we worked within the capabilities of our electronic record system. It is therefore limited to this specific setting. However, features of the methodology of this project are generalisable, for example, working with systems already in place and consulting stakeholders before the intervention. This feature both raises awareness and gives stakeholders a sense of ownership in the project. This feature could create bias, in the sense that 
the same doctors were surveyed before and after implementation, and they thus had invested in the intervention to a small extent. It would, however, serve to reduce variability of surveying a different group of junior doctors.

The capture rates were low in Lambeth, and it would be useful to test the hypothesis above: that the motivation of the ward doctors on triage is key to capture rates and that motivation depends on level of involvement in the project which then leads to accountability.

\section{CONCLUSION}

This project implemented a succinct handover system which was easy to implement, well received among medical colleagues and sustainable over a long time period. The results show that the lack of a formal handover process was a concern shared by doctors, and our intervention was considered an effective way of addressing this. The fact that the majority of patients received a handover in two of the boroughs shows that the intervention was workable and could achieve good capture rates. The measures we employed were appropriate and gave a subjective measure of utility. It is likely that for a small investment in time from the triage ward doctor writing the handover, time-consuming referrals and costly blood tests would not have been duplicated on locality wards.

The output of the project-namely, the increased satisfaction that junior doctors had with the handover process-is valuable, as this was who the project was designed for. Creating a system by doctors for doctors that they feel really works, in consultation with them and partly designed by them, increases morale and promotes sustainability. In terms of sustainability, we have data 1 year on showing good capture rates. There was no communication from the authors to the doctors working on the ward during the time of our reaudit or during the previous year. The intervention was sustained solely due to it being present in the Junior Doctor's Guide and due to the continuity of senior support for the project. There is no reason to doubt the intervention will be sustained for years to come, as it has become an expected part of the work of a doctor working on Lewisham triage ward. We also showed how it is possible to roll-out projects across large trusts that contain several sites. We were able to make the case for the utility and worth of the project to the two junior doctorss who agreed to undertake replica projects at Lambeth and Croydon. Although there are variations between working practices and computing systems between mental health trusts, it is likely that our improvement could be replicated in any psychiatric hospital that uses the triage model.

Acknowledgements Dr Chun Chiang Sin Fai Lam for his helpful comments and advice on this project.

Contributors $A D$ and TR designed the study. RR supervised the study. AD, TR, LB and $\mathrm{MD}$ collected data. $\mathrm{AD}$ wrote the manuscript. All authors contributed to the critical revision of the manuscript.

Competing interests None declared.

Ethics approval The Psychological Medicine Clinical and Academic Group Audit Committee at South London and the Maudsley NHS Foundation Trust had clinical governance oversight of the project. As the work was deemed an improvement study and not a study on human subjects, ethical approval was not required, as per local policy.

Provenance and peer review Not commissioned; externally peer reviewed.

Open Access This is an Open Access article distributed in accordance with the Creative Commons Attribution Non Commercial (CC BY-NC 4.0) license, which permits others to distribute, remix, adapt, build upon this work non-commercially, and license their derivative works on different terms, provided the original work is properly cited and the use is non-commercial. See: http://creativecommons.org/ licenses/by-nc/4.0/

(c) Published by the BMJ Publishing Group Limited. For permission to use (where not already granted under a licence) please go to http://www.bmj.com/company/ products-services/rights-and-licensing/

\section{REFERENCES}

1. British Medical Association. Safe handover: safe patients. Guidance on clinical handover for clinicians and managers. London: BMA, 2004.

2. General Medical Council. Good Medical practice. London: General Medical Council, 2013.

3. Royal College of Physicians. Acute care toolkit 1: handover. London: RCP, 2011.

4. The Royal College of Surgeons of England. Safe handover: guidance from the working time directive working party. London: RCS, 2007.

5. Boyer M, Tappenden J, Peter M. Improving weekend out of hours surgical handover (WOOSH). BMJ Qual Improv Rep 2016;5:1.

6. Walton $\mathrm{H}$, Munro W. Improving the quality of handover by addressing handover culture and introducing a new, multi-disciplinary, teambased handover meeting. BMJ Qual Improv Rep 2015;4:u206069. w2989.

7. Messing J. Improving handover from intensive care to ward medical teams with simple changes to paperwork. BMJ Qual Improv Rep 2015;4:u206467.w2913.

8. Parbhoo A, Batte J. Traffic lights: putting a stop to unsafe patient transfers. BMJ Qual Improv Rep 2015;4:u204799.w2079.

9. Williams P, Csipke E, Rose D, et al. Efficacy of a triage system to reduce length of hospital stay. Br J Psychiatry 2014;204:480-5.

10. Walton $\mathrm{H}$, Munro $\mathrm{W}$. Improving the quality of handover by addressing handover culture and introducing a new, multi-disciplinary, teambased handover meeting. BMJ Qual Improv Rep 2015;4:u206069. w2989.

11. Ashton C. Improving weekend patient handover. BMJ Qual Improv Rep 2014;2.

12. Timmons $\mathrm{S}$, Baxendale $\mathrm{B}$, Buttery $\mathrm{A}$, et al. Implementing human factors in clinical practice. Emerg Med J 2015;32.

13. Till A, Sall H, Wilkinson J. Safe handover: safe patients - the electronic handover system. BMJ Qual Improv Rep 2014;2:u202926. w1359. 